\title{
Level of invasion into fibromuscular band is an independent factor for positive surgical margin and biochemical recurrence in men with organ confined prostate cancer
}

Aram Kim", Myong Kim¹, Se Un Jeong ${ }^{2}$, Cheryn Song ${ }^{1}$, Yong Mee Cho ${ }^{2}$, Jae Yoon Ro ${ }^{3}$ and Hanjong Ahn ${ }^{1 *}$

\begin{abstract}
Background: This study aimed investigate the effect of the level of invasion into fibromuscular band (FMB) of prostate on the positive surgical margin (PSM) and biochemical recurrence (BCR) after radical prostatectomy (RP) in patients with organ-confined (pT2) prostate cancer.

Methods: The clinical and pathological data of 461 consecutive patients with pT2 prostate cancer were evaluated regarding the level of invasion into FMB. The relationship between levels of invasion into FMB and PSM / BCR was assessed.

Results: The rate of PSM at an FMB level of at 2 was $18.8 \%$, which was significantly greater than the rates at levels $0(5.4 \%)$ and $1(7.8 \%)$. The level of FMB $(p=0.007)$ and percentage of tumor volume $(p=0.012)$ were identified as independent factors predictive of a positive surgical margin in a multivariate analysis. The 5 -year BCR-free survival rates for a level 0-1 FMB with negative surgical margin, level 0-1 FMB with positive surgical margin, level 2 FMB with negative surgical margin, and level 2 FMB with positive surgical margin were $96.6 \%, 86.4 \%, 85.6 \%$, and $72.9 \%$, respectively $(p<0.001)$. A level 2 FMB $(p=0.050)$, positive surgical margin $(p=0.001)$, and surgical Gleason score $(p=0.001)$ were identified as independent predictors of a BCR of pT2 prostate cancer.

Conclusions: Among patients with negative surgical margins, the surgical Gleason score and level of FMB independently affected the incidence of a BCR of pT2 prostate cancer. The level of FMB was an independent predictor of both a positive surgical margin and a BCR of pT2 disease. Accordingly, the level of FMB might help to further stratify the prognosis of patients with pT2 disease.
\end{abstract}

Keywords: Organ confined prostate cancer, Biochemical recurrence, Positive surgical margin, Fibromuscular band, Predictive factor

\section{Background}

All oncologic surgeries aim to completely remove cancers. Therefore, the presence of a positive surgical margin (PSM) after radical prostatectomy (RP) for prostate cancer is considered an adverse event in curing this cancer, with outcome associated with prostate specific antigen (PSA) biochemical recurrence (BCR) and poor outcome $[1,2]$. The reported rates of PSMs among

\footnotetext{
*Correspondence: hjahn@amc.seoul.kr

'Department of Urology, Asan Medical Center, University of Ulsan College of Medicine, 88 Olympic-Ro 43 Gil Songpa-Gu, Seoul 05505, Republic of Korea

Full list of author information is available at the end of the article
}

pathologically localized prostatectomy specimens vary from $6.5 \%$ to $38 \%$ in contemporary series of RP $[1,3]$. The rate of PSM for pT2 prostate cancers is thought to reflect the surgeon's experience $[4,5]$. However, tumorbehavioral factors might also influence the rate of PSM for organ-confined prostate cancer. A large-volume tumor adjacent to the prostatic capsule may be prone to a PSM if the surgeon rigorously attempts to preserve the nerves and maximize the remaining functioning urethra. The level of prostatic capsular invasion, which focuses on the extra-prostatic extension (EPE), was reported to affect the incidence of BCR [6]. However, recent articles 
have avoided the term "prostatic capsule" [6-8], as the prostate does not have a true capsule at the apex, anterior side, and base. Therefore, we instead revisited the level of invasion into fibromuscular band (FMB) as an independent factor for a PSM or BCR in patients with organ-confined prostate cancer. We hypothesized that the level of invasion into FMB would be an independent tumor-behavioral factor that could affect the rates of PSM and BCR in patients with PT2 prostate cancer.

\section{Methods}

\section{Patient selection}

We reviewed 473 patients with pT2 prostate cancer who had undergone RP (robotic: 369, open: 104) at our hospital from January 2010 to March 2014. We excluded four patients who received neoadjuvant treatment and eight patients for whom complete clinical data were unavailable or who were lost to follow-up. Eventually, 461 patients were enrolled, with a median follow-up of 51.2 (range: 1.9-74.9) months. The serum PSA levels were measured at 3-month intervals during the first year after $\mathrm{RP}$, 6-month intervals during years $2-5$, and annually thereafter. A BCR was defined as a PSA level $>0.2 \mathrm{ng} / \mathrm{mL}$.

\section{Pathologic evaluation and determination of the level of invasion into fibromuscular band}

All surgical specimens were assessed microscopically after histological sectioning. Each specimen was weighed, and the external surface was covered in India ink prior to fixation in $10 \%$ formalin. Each specimen was examined in 3-5 $\mathrm{mm}$ sections from the base to the apex, perpendicular to the major. Subsequently, the sections were divided into halves or quadrants to fit the cassettes routinely used for paraffin embedding, and slide-mounted thin sections were stained with hematoxylin-eosin. Primary and secondary
Gleason scores were assigned to the total cancer within the specimen, according to the revised (2005) criteria for Gleason scoring [9]. Each specimen was staged according to the 2010 American Joint Committee on Cancer staging system [10]. The presence of cancer cells in the inked surface of RP specimen was considered a positive surgical margin [11]. The percentage of tumor volume (PTV) was estimated by the sum of visually determined tumor foci relative to the prostate gland on every section, and categorized into three groups: $<5 \%, 5-15.0 \%$, and $>15.0 \%$. The levels of EPE were classified as 0,1 , and 2 according to the classification by Wheeler et al. (Fig. 1) [12]. In level 0, the cancer cells are located in the prostatic stroma with a normal gland. Level 1 involves cancer cells within the prostatic stroma but beyond the boundary of normal gland. Level 2 involves cancer cells confined to the prostate, within a layer more fibrous than muscular. We used the highest level of FMB if there were several tumors in prostate and the levels of FMB were evaluated and confirmed by 2 pathologists.

\section{Statistical analysis}

The patients' clinical and pathological characteristics were compared according to the levels of EPE, using the $\chi^{2}$ test (categorical variables) and Student's $t$-test (continuous variables). Uni- and multivariate binary logistic regression analyses were performed to determine the pre- and post-operative variables predictive of a PSM. The 5-year BCR-free survival rate after RP was estimated using the Kaplan-Meier method. Cox proportional hazard analyses were performed to identify the prognostic factors affecting the incidence of BCR. All statistical analyses were performed using commercially available software $\left(\mathrm{SPSS}^{\circ}\right.$ version 21.0, IBM, Chicago, IL, USA), and a $p$ value $<0.05$ was considered statistically significant.

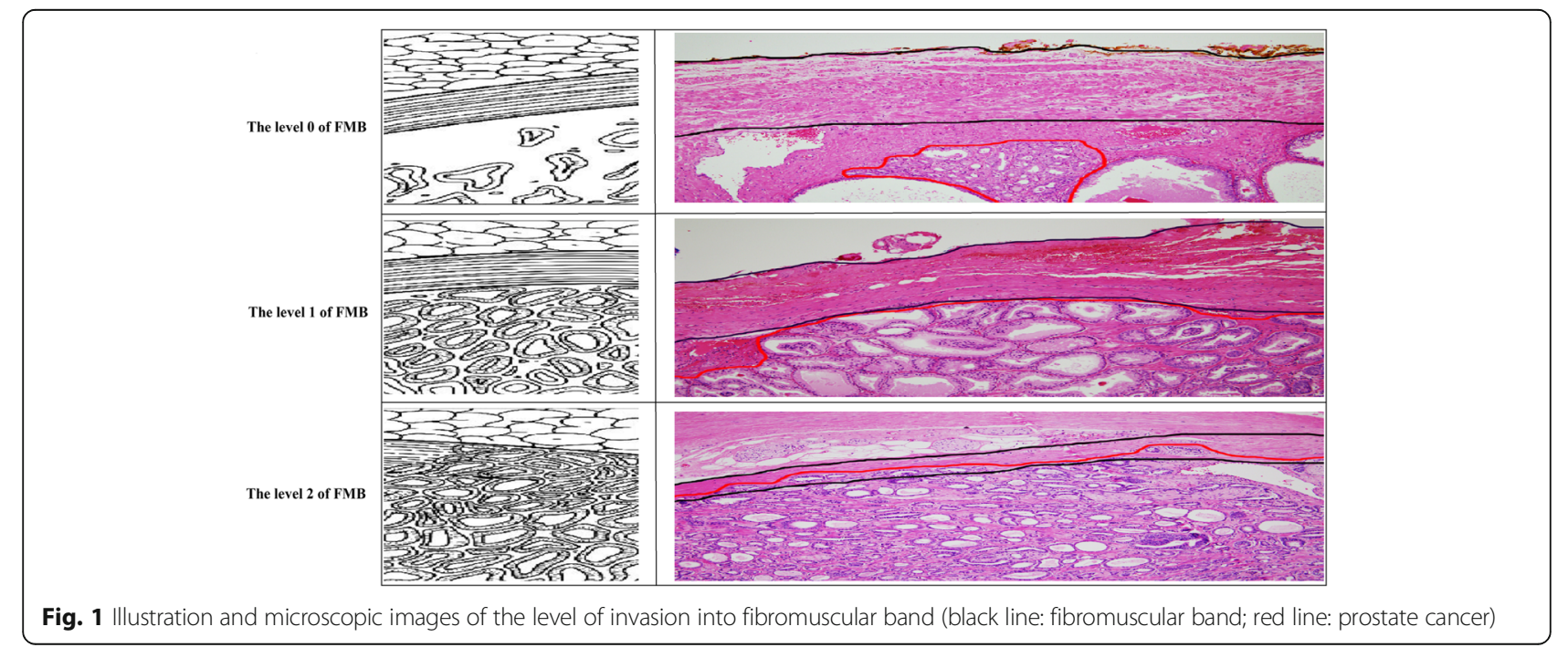




\section{Results}

\section{Comparison of clinical features according to the level of} invasion into fibromuscular band

The patients' clinical and pathological characteristics are summarized in Table 1. A higher clinical T stage, percentage of positive cores, and biopsy Gleason score were more frequently observed among patients with level 2 FMB, compared to those with levels 0 and 1 . The PTV increased significantly according to the level of FMB, and higher surgical Gleason scores were more frequent with a level 2 FMB than with a level 0 or 1 FMB (all $p<0.001)$. Other parameters such as the PSA and prostate size did not differ significantly among the three groups ( $\mathrm{p}$ range, $0.426-0.672$; Table 1 ).

\section{Associations between the level of invasion into fibromuscular band and PSM after RP}

The rates of PSM at a level 2 FMB (18.7\%) were significantly greater than those at levels $0(5.4 \%)$ and $1(7.8 \%)(p=0.001)$, whereas this parameter did not differ significantly between levels 0 and $1(p=0.495$; Table 1). In a univariate analysis with postoperative variables, the PTV $(\mathrm{p}=0.001)$ and level of FMB $(p=0.001)$ were significant factors affecting the PSM. The PTV $(p=0.012)$ and level of EPE $(p=0.007)$ were also identified as independent factors in a multivariate analysis (Table 2).
Associations between the level of invasion into fibromuscular band and BCR-free survival after RP

During the follow-up period, 29 patients (6.2\%) experienced a BCR at a mean interval of 28.8 months after RP. Overall, the 5-year BCR-free survival rate was $92.4 \%$. The 5-year BCR-free survival rates of patients with level 0-1 FMB and level 2 FMB were $95.4 \%$ and $84.2 \%$, respectively ( $p=0.002$; Fig. $2 \mathrm{a}$ ), and those of patients with a negative surgical margin and PSM were $94.4 \%$ and $78.5 \%$, respectively ( $p<0.001$; Fig. $2 \mathrm{~b}$ ). The 5 -year BCRfree survival rates of patients with Gleason scores of 6 , $3+4,4+3$ and $8-10$ were $97.6 \%, 94.5 \%, 86.7 \%$, and $76.6 \%$, respectively $(\mathrm{p}<0.001)$.

When patients were stratified by margin status and level of FMB (level 0-1 vs. level 2), the 5-year BCR-free survival rates of those with a level $0-1$ FMB and negative surgical margin, level $0-1$ FMB and PSM, level 2 FMB and negative surgical margin, and level 2 FMB and PSM were $96.6 \%, 86.4 \%, 85.6 \%$, and $72.9 \%$, respectively $(p<0.001$; Fig. 2c). Patients with a level $0-1$ FMB and PSM did not significantly differ from those with a level 2 FMB and negative surgical margin in terms of survival $(p=0.578)$. Among the subgroups with surgical Gleason scores greater than $4+3$, the corresponding survival rates were $88.4 \%, 87.2 \%, 76.2 \%$, and $53.3 \%$, respectively ( $p=0.012$; Fig. $2 \mathrm{~d})$.

A multivariate analysis of postoperative variables predictive of a BCR identified the surgical Gleason

Table 1 Clinical and pathological characteristics of the patients with pT2 prostate cancer

\begin{tabular}{|c|c|c|c|c|c|}
\hline Level of FMB & Total & Level 0 & Level 1 & Level 2 & $p$ value \\
\hline No. of patients & 461 & 74 & 243 & 144 & \\
\hline Mean PSA (ng/mL) & 6.6 & 6.5 & 6.5 & 7.0 & 0.672 \\
\hline Prostate size (cc) & 36.0 & 37.8 & 35.2 & 36.5 & 0.426 \\
\hline Mean \% of positive biopsy cores & 20.2 & 13.0 & 19.0 & 26.2 & $<0.001$ \\
\hline Clinical stage & & & & & 0.031 \\
\hline CT1 & 257 & 53 & 130 & 73 & \\
\hline$\geq \mathrm{cT} 2$ & 204 & 21 & 113 & 71 & \\
\hline Biopsy Gleason score, No. & & & & & $<0.001$ \\
\hline 6 & 197 & 50 & 104 & 43 & \\
\hline $3+4$ & 160 & 19 & 84 & 57 & \\
\hline $4+3$ & 71 & 2 & 38 & 31 & \\
\hline $8-10$ & 33 & 3 & 17 & 13 & \\
\hline Surgical Gleason score, No. & & & & & $<0.001$ \\
\hline 6 & 124 & 41 & 65 & 18 & \\
\hline $3+4$ & 205 & 26 & 110 & 69 & \\
\hline $4+3$ & 99 & 4 & 51 & 44 & \\
\hline $8-10$ & 33 & 3 & 17 & 13 & \\
\hline Mean PTV & 8.7 & 3.5 & 8.4 & 11.5 & $<0.001$ \\
\hline Rate of PSM, N (\%) & $50(10.8 \%)$ & $4(5.4 \%)$ & $19(7.8 \%)$ & $27(18.7 \%)$ & 0.001 \\
\hline
\end{tabular}

FMB fibromuscular band, PSA prostate specific antigen, PTV percentage of tumor volume, PSM positive surgical margin 
Table 2 Risk factors for PSM after radical prostatectomy in pT2 prostate cancer

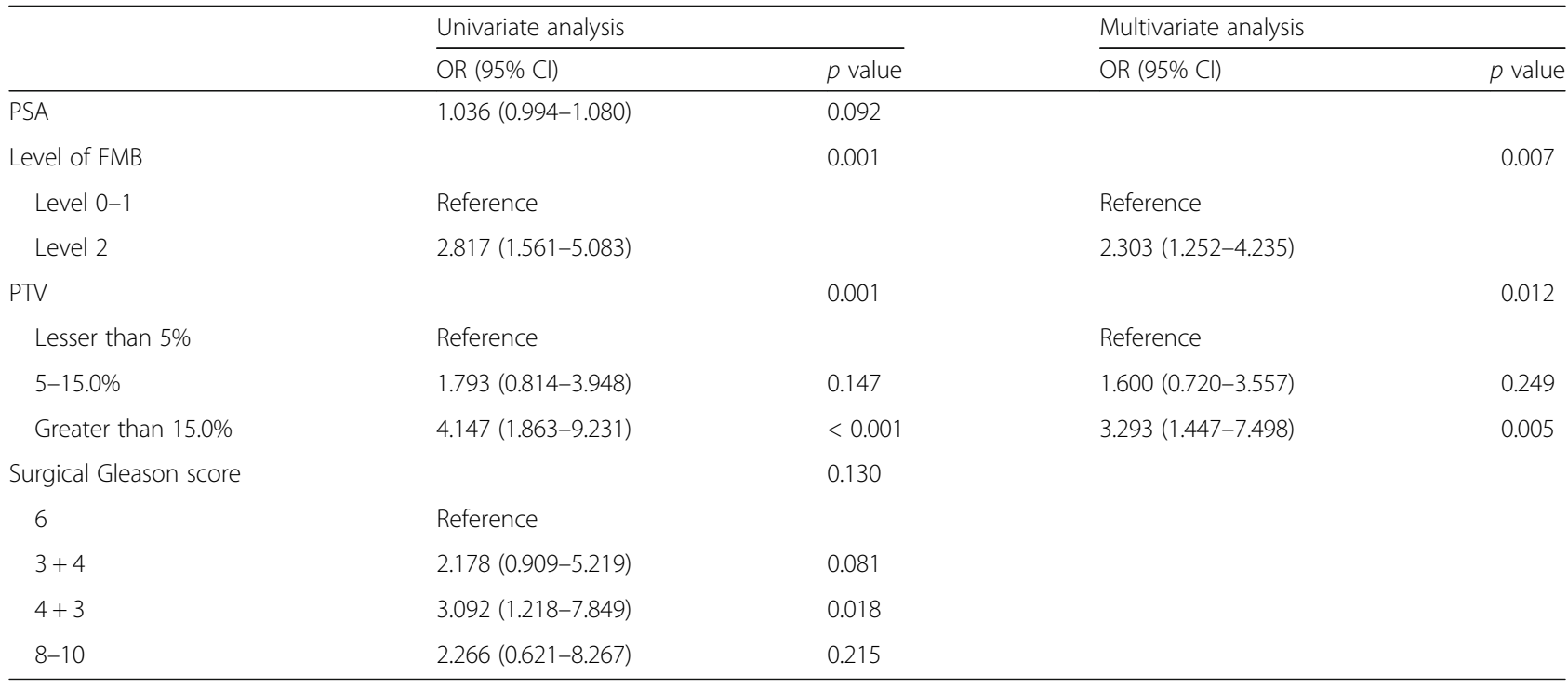

PSA prostate specific antigen, FMB fibromuscular band, PTV percentage of tumor volume, PSM positive surgical margin
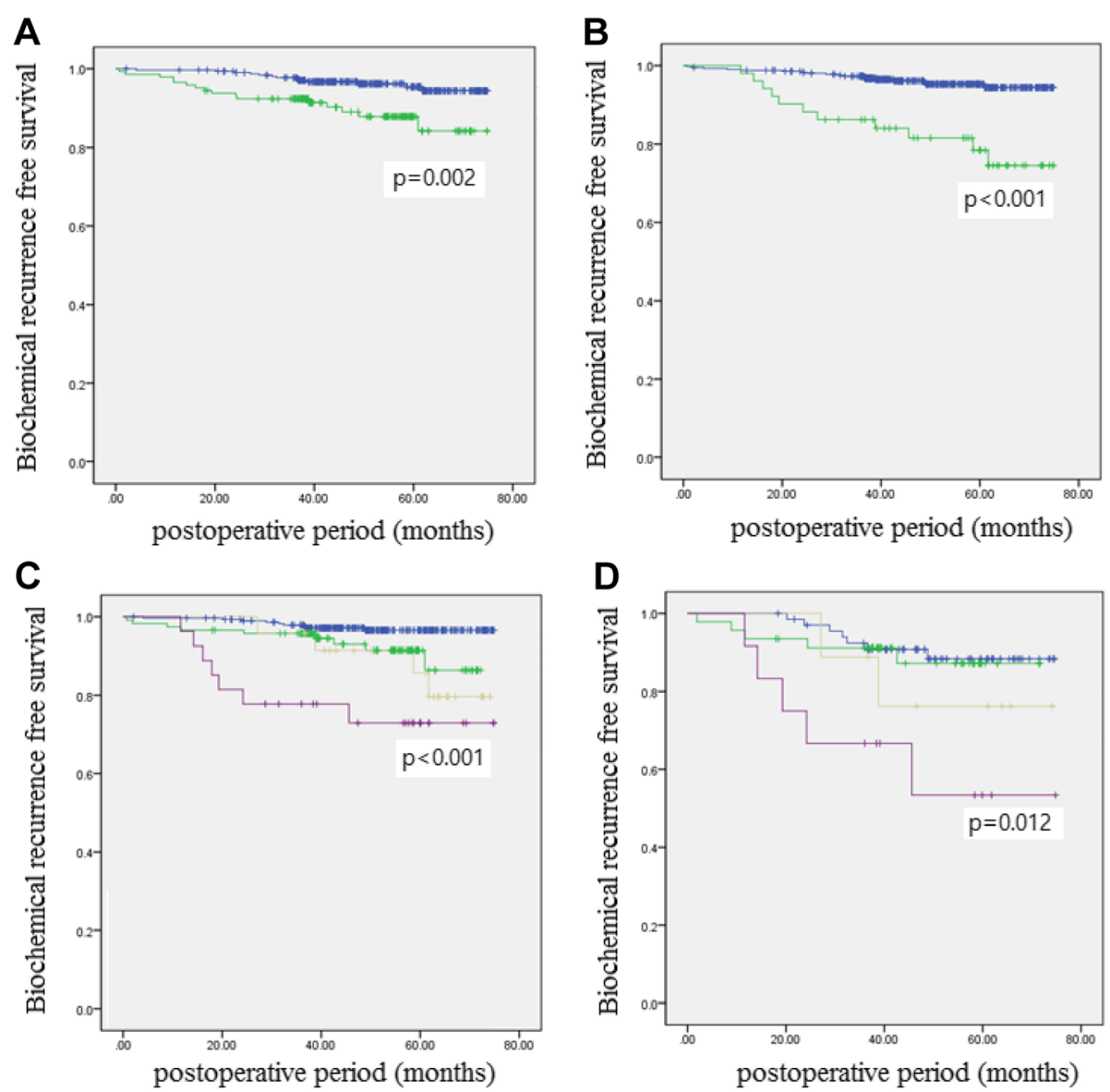

Fig. 2 Biochemical recurrence-free survival over time in patients with pT2 prostate cancer and (a) a level 0-1 FMB (Blue) vs. level 2 FMB (Green); b negative surgical margin (Blue) vs. positive surgical margin (Green); c level 0-1 FMB and NSM (Blue) vs. level 0-1 FMB and PSM (Green) vs. level 2 FMB and NSM (Yellow) vs. level 2 FMB and PSM (Purple); d Among patients with a surgical Gleason score $>4+3$, a level 0-1 FMB and NSM (Blue) vs. level 0-1 FMB and PSM (Green) vs. level 2 FMB and NSM (Yellow) vs. level 2 FMB and PSM (Purple) 
score $(p=0.001), \operatorname{PSM}(\mathrm{p}=0.001)$, and level 2 FMB $(p=0.050)$ as independent predictors (Table 3). Among the subgroups with negative surgical margins, the level of FMB remained an independent prognostic factor for a BCR $(p=0.045)$, along with the surgical Gleason score $(\mathrm{p}=0.001)$ and PSA level $(p<0.001)$.

\section{Discussion}

Although the effect of a PSM on long-term cancerrelated mortality remains controversial [2, 13], this factor has been proposed as an important predictor of disease progression $[14,15]$ and one of the strongest determinants of the possibility of benefitting from adjuvant radiotherapy [1]. Among pT2 prostate cancers, a PSM has been considered an iatrogenic factor because these tumors are confined to the prostate; therefore, the surgeons' experience might be important for reducing the incidence of PSM in a prostate-confined cancer [4, 5]. Previous studies have reported dramatically different rates of PSM according to the surgeons' clinical experience [16], and found that the learning curves for surgical margins after open or laparoscopic RP plateaued at approximately 200-250 cases $[17,18]$. We also reported a similar experience regarding the learning curves for surgical margins in a robotic RP series, wherein we demonstrated that the robotic RP reached a comparable PSM rate for pT3 disease with a surgical experience exceeding 500 cases [19]. However, even after transcending this learning curve for surgical margins, surgeons are among organ-confined prostate disease. Tumor-behavioral factors, such as the extent of EPE, have been identified as risk factors for a PSM, along with the preoperative PSA level and Gleason score [4, 20]. However, it remains unclear whether the tumorbehavioral factor itself is important in terms of the PSM. We previously identified the PTV as an independent risk factor for a PSM $(p=0.035)$, and both the PTV $(p<0.001)$ and surgical Gleason score $(\geq 8 ; p=0.021)$ as independent predictors of a BCR of organ-confined prostate cancer [21].

In this study, we hypothesized that the level of invasion into FMB might be another important tumorbehavioral factor associated with the risks of PSM and BCR after a RP of organ-confined disease. Our current results demonstrated that the level of invasion into FMB was an independent risk factor for a PSM (level 2 PCI; $p=0.007)$, along with the tumor volume $(p=0.013$; Table 2). Accordingly, the level of invasion into FMB, tumor volume, and surgical Gleason score are tumorbehavioral factors that might promote a PSM after RP for organ-confined prostate cancer. On the whole, these results agree with those in our previous reports [21]. However, the significance of PTV for BCR was not consistent with our previous study, which included an older cohort (2000-2007) and used specialized method of PTV estimation [21]. The mean PTV was higher in our previous study than in the present study (12.1\% vs. $8.7 \%)$.

We believe that the level of invasion into FMB has important clinical implications for organ-confined prostate cancer. Although a previous study reported that higher levels of prostatic capsular invasion were associated with a more adverse prognosis among patients with prostate

Table 3 Risk factors for BCR after radical prostatectomy (RP) in pT2 prostate cancer

\begin{tabular}{|c|c|c|c|c|}
\hline & \multicolumn{2}{|l|}{ Univariate analysis } & \multicolumn{2}{|l|}{ Multivariate analysis } \\
\hline & HR $(95 \% \mathrm{Cl})$ & $\overline{p \text { value }}$ & HR (95\% Cl) & $p$ value \\
\hline Level of FMB & & 0.003 & & 0.050 \\
\hline Level 0-1 & Reference & & Reference & \\
\hline Level 2 & $3.067(1.471-6.393)$ & & $2.124(1.001-4.508)$ & \\
\hline Surgical Gleason score & & $<0.001$ & & 0.001 \\
\hline 6 & Reference & & Reference & \\
\hline $3+4$ & 2.417 (0.1513-11.383) & 0.264 & $1.878(0.395-8.923)$ & 0.428 \\
\hline $4+3$ & 7.909 (1.770-35.348) & 0.007 & 5.585 (1.229-25.384) & 0.026 \\
\hline $8-10$ & 14.099 (2.929-67.876) & 0.001 & $10.311(2.119-50.175)$ & 0.004 \\
\hline PTV & & 0.084 & & \\
\hline Lesser than $5 \%$ & Reference & & & \\
\hline $5-15.0 \%$ & $2.421(0.872-6.723)$ & 0.090 & & \\
\hline Greater than $15.0 \%$ & $3.364(1.150-9.841)$ & 0.027 & & \\
\hline Surgical Margin Status & & $<0.001$ & & 0.001 \\
\hline Negative & Reference & & Reference & \\
\hline Positive & $4.813(2.268-10.213)$ & & $3.607(1.683-7.726)$ & \\
\hline
\end{tabular}

$B C R$ biochemical recurrence, $F M B$ fibromuscular band, $P T V$ percentage of tumor volume 
cancer, the authors did not demonstrate whether the level of prostatic capsular invasion was an independent prognostic factor for a BCR of pT2 prostate cancer [6]. In our study, we used the term "fibromuscular band (FMB)" which was commonly accepted instead of "prostatic capsule" [6] and identified the level of invasion into FMB as an independent predictor of a BCR (Table 3). The 5-year BCR-free survival rate among patients with a level $2 \mathrm{FMB}$ and negative surgical margin was similar to that of patients with a level 0-1 FMB group and PSM (86.4\% vs. $85.6 \%$; $p=0.578$; Fig. 2c). Especially, patients with pT2 disease, a level 2 FMB, PSM, and a surgical Gleason score $\geq 4+3$ had a worse 5 -year BCR-free survival rate, compared to patients with a level 0-1 EPE, PSM, and surgical Gleason score $\geq 4+3$, although this difference failed to reach statistical significance $(53.3 \%$ vs. $76.2 \% ; p=0.225)$. The recent American Urology Association (AUA)/American Society for Therapeutic Radiology and Oncology (ASTRO) guideline mandated the use of adjuvant radiation therapy for patients with pT3 prostate cancer, based on the results from several randomized controlled trials [22]. Moreover, several studies of non-organ confined prostate cancer demonstrated that adjuvant radiotherapy significantly reduced the risk of $\mathrm{BCR}$ and improved metastasis-free and overall survival [23-25]. Although RP provides decent overall long-term oncologic control and favorable survival rates in patients with organ-confined prostate cancer, we note that the clinical courses might vary depending on the presence of other risk factors. Given the BCR-free survival rates reported for the control arms of previous studies [23-25], we believe that a certain subgroup of patients with pT2 disease will require adjuvant radiotherapy to reduce the risk of BCR after RP. The group with the worst prognosis in the present study, those with pT2 disease, a level 2 FMB, PSM, and surgical Gleason score $\geq 4+3$, had a 5-year BCR-free survival rate of $53.3 \%$, similar to that of patients with T3b disease in a previous study [26]. We believe that these patients should therefore be considered candidates for adjuvant radiotherapy after RP.

The present study had a retrospective design and relatively small sample size, and there were other limitations, such as a critical reproducibility of the level of invasion into FMB. Pathologists must re-evaluate the level of invasion into FMB, as well as the levels of all tumors. The highest level of FMB should be used for cases involving multiple tumors in the prostate. Furthermore, the effect of the level of invasion into FMB according to the tumor location remains controversial, and additional studies are needed. Despite these limitations, our study attempted to revisit the concept of the level of invasion into FMB as a tumor-behavioral factor in a contemporary RP cohort. External validation studies with larger cohorts or prospectively designed studies are needed to confirm the clinical implications of our findings.

\section{Conclusions}

The level of invasion into FMB is an independent tumor-behavioral factor for PSM and BCR in organconfined prostate cancer. Patients with a level 2 FMB had a higher risk of BCR after RP. Therefore, the level of invasion into FMB might be used to stratify patients with pT2 disease and a poor prognosis.

\section{Abbreviations \\ Acknowledgments \\ Not applicable \\ Funding \\ No fund \\ Availability of data and materials \\ Please contact author for data requests.}

BCR: Biochemical recurrence; EPE: Extra-prostatic extension;

FMB: Fibromuscular band; PSA: Prostate specific antigen; PSM: Positive

surgical margin; PTV: Percentage of tumor volume; RP: Radical prostatectomy

\section{Authors' contributions}

AK collected data, design of the study, analysis interpretation of data and drafted the manuscript. MK and CS contributed to surgeries, the conception and design of the study, SJ contributed to acquisition and analysis of pathologic data and interpretation. YC and JR contributed to slide review and data analysis. All authors read and approved the final manuscript. HA contributed to Study design, data interpretation, drafting the manuscript, final approval of the version to be published.

Ethics approval and consent to participate

The study was approved by the Ethics Committee of Asan Medical Center, University of Ulsan College of Medicine (2014-0980).

Consent for publication

Not applicable

Competing interests

The authors declare that they have no competing interests.

\section{Publisher's Note}

Springer Nature remains neutral with regard to jurisdictional claims in published maps and institutional affiliations.

\section{Author details}

${ }^{1}$ Department of Urology, Asan Medical Center, University of Ulsan College of Medicine, 88 Olympic-Ro 43 Gil Songpa-Gu, Seoul 05505, Republic of Korea. ${ }^{2}$ Department of Pathology, Asan Medical Center, University of Ulsan College of Medicine, Seoul 05505, Republic of Korea. ${ }^{3}$ Department of Pathology and Genomic Medicine, Houston Methodist Hospital, Weill Medical College of Cornell University, Houston, TX 10065, USA. ${ }^{4}$ Department of Urology, Konkuk University Medical Center, Konkuk University School of Medicine, Seoul 05030, Republic of Korea.

Received: 2 October 2017 Accepted: 24 January 2018

Published online: 02 February 2018

\section{References}

1. Yossepowitch $\mathrm{O}$, et al. Positive surgical margins after radical prostatectomy: a systematic review and contemporary update. Eur Urol. 2014;65(2):303-13.

2. Chalfin $\mathrm{HJ}$, et al. Impact of surgical margin status on prostate-cancer-specific mortality. BJU Int. 2012;110(11):1684-9. 
3. Yossepowitch $\mathrm{O}$, et al. Positive surgical margins in radical prostatectomy: outlining the problem and its long-term consequences. Eur Urol. 2009;55(1): 87-99.

4. Eastham JA, et al. Variations among individual surgeons in the rate of positive surgical margins in radical prostatectomy specimens. J Urol. 2003; 170(6 Pt 1):2292-5.

5. Chun FK, et al. Surgical volume is related to the rate of positive surgical margins at radical prostatectomy in European patients. BJU Int. 2006;98(6): 1204-9.

6. Wheeler TM, et al. Clinical and pathological significance of the level and extent of capsular invasion in clinical stage T1-2 prostate cancer. Hum Pathol. 1998;29(8):856-62.

7. Ayala AG, et al. The prostatic capsule: does it exist? Its importance in the staging and treatment of prostatic carcinoma. Am J Surg Pathol. 1989;13(1):21-7.

8. Paluru S, Epstein J. Does the distance between tumor and margin in radical prostatectomy specimens correlate with prognosis: relation to tumor location. Hum Pathol. 2016;56:11-5.

9. Epstein Jl, et al. The 2005 International Society of Urological Pathology (ISUP) consensus conference on Gleason grading of prostatic carcinoma. Am J Surg Pathol. 2005;29(9):1228-42.

10. Edge, S.B., et al., Prostate, in AJCC cancer staging manual, American joint committee on Cancer, Editor. 2010, Springer: New York. p. 457-468.

11. Epstein Jl, et al. Prognostic factors and reporting of prostate carcinoma in radical prostatectomy and pelvic lymphadenectomy specimens. Scand J Urol Nephrol Suppl. 2005;216:34-63.

12. Wheeler TM. Anatomic considerations in carcinoma of the prostate. Urol Clin North Am. 1989;16(4):623-34.

13. Stephenson AJ, et al. Do margins matter? The influence of positive surgical margins on prostate cancer-specific mortality. Eur Urol. 2014;65(4):675-80.

14. Stephenson AJ, et al. Location, extent and number of positive surgical margins do not improve accuracy of predicting prostate cancer recurrence after radical prostatectomy. J Urol. 2009;182(4):1357-63.

15. Eastham JA, et al. Prognostic significance of location of positive margins in radical prostatectomy specimens. Urology. 2007;70(5):965-9.

16. Roder MA, et al. Risk factors associated with positive surgical margins following radical prostatectomy for clinically localized prostate cancer: can nerve-sparing surgery increase the risk? Scand J Urol. 2014:48(1):15-20.

17. Secin FP, et al. The learning curve for laparoscopic radical prostatectomy: an international multicenter study. J Urol. 2010;184(6):2291-6.

18. Vickers $A$, et al. The learning curve for surgical margins after open radical prostatectomy: implications for margin status as an oncological end point. J Urol. 2010;183(4):1360-5.

19. Park J, et al. Comparison of oncological outcomes between retropubic radical prostatectomy and robot-assisted radical prostatectomy: an analysis stratified by surgical experience. World J Urol. 2014;32(1):193-9.

20. Wieder JA, Soloway MS. Incidence, etiology, location, prevention and treatment of positive surgical margins after radical prostatectomy for prostate cancer. J Urol. 1998;160(2):299-315.

21. Song C, et al. Tumor volume, surgical margin, and the risk of biochemical recurrence in men with organ-confined prostate cancer. Urol Oncol. 2013; 31(2):168-74.

22. Wiegel T, et al. Adjuvant radiotherapy versus wait-and-see after radical prostatectomy: 10-year follow-up of the ARO 96-02/AUO AP 09/95 trial. Eur Urol. 2014;66(2):243-50

23. Bolla M, et al. Postoperative radiotherapy after radical prostatectomy for high-risk prostate cancer: long-term results of a randomised controlled trial (EORTC trial 22911). Lancet. 2012;380(9858):2018-27.

24. Thompson IM, et al. Adjuvant radiotherapy for pathological T3NOMO prostate cancer significantly reduces risk of metastases and improves survival: long-term followup of a randomized clinical trial. J Urol. 2009; 181(3):956-62.

25. Wiegel T, et al. Phase III postoperative adjuvant radiotherapy after radical prostatectomy compared with radical prostatectomy alone in pT3 prostate cancer with postoperative undetectable prostate-specific antigen: ARO 96-02/AUO AP 09/95. J Clin Oncol. 2009;27(18):2924-30.

26. Steuber T, et al. Comparative assessment of the 1992 and 2002 pathologic T3 substages for the prediction of biochemical recurrence after radical prostatectomy. Cancer. 2006;106(4):775-82.

\section{Submit your next manuscript to BioMed Central and we will help you at every step:}

- We accept pre-submission inquiries

- Our selector tool helps you to find the most relevant journal

- We provide round the clock customer support

- Convenient online submission

- Thorough peer review

- Inclusion in PubMed and all major indexing services

- Maximum visibility for your research

Submit your manuscript at www.biomedcentral.com/submit
) Biomed Central 